\title{
An eye-tracking study of feature-based choice in one-shot games
}

\author{
Giovanna Devetag • Sibilla Di Guida $\cdot$ Luca Polonio
}

Received: 28 March 2013/Revised: 18 February 2015/Accepted: 20 February 2015/

Published online: 26 February 2015

(C) Economic Science Association 2015

\begin{abstract}
Previous experimental research suggests that individuals apply rules of thumb to a simplified mental model of the "real" decision problem. We claim that this simplification is obtained either by neglecting the other players' incentives and beliefs or by taking them into consideration only for a subset of game outcomes. We analyze subjects' eye movements while playing a series of two-person, $3 \times 3$ oneshot games in normal form. Games within each class differ by a set of descriptive features (i.e., features that can be changed without altering the game equilibrium properties). Data show that subjects on average perform partial or non-strategic analysis of the payoff matrix, often ignoring the opponents payoffs and rarely performing the necessary steps to detect dominance. Our analysis of eye-movements supports the hypothesis that subjects use simple decision rules such as "choose the
\end{abstract}

Electronic supplementary material The online version of this article (doi:10.1007/s10683-015-94325) contains supplementary material, which is available to authorized users.

\section{G. Devetag}

Department of Business and Management, Luiss Guido Carli, Viale Pola 12, 00198 Rome, Italy e-mail: giovanna.devetag@gmail.com

\section{S. Di Guida $(\bowtie)$}

Department of Business and Economics, COHERE, Syddansk Universitet, Campusvej 55, 5230 Odense M, Denmark

e-mail: sidg@sam.sdu.dk

\section{S. Di Guida}

SBS-EM, ECARES, Université Libre de Bruxelles, Brussels, Belgium

\section{Polonio}

Department of Cognitive Science and Education, University of Trento, Corso Bettini 31, 38068 Rovereto, Italy

e-mail: luca.polonio@unitn.it

L. Polonio

Center for Mind and Brain Sciences, University of Trento, Corso Bettini 31, 38068 Rovereto, Italy 
strategy with the highest average payoff" or "choose the strategy leading to an attractive and symmetric outcome" without (optimally) incorporating knowledge on the opponent's behavior. Lookup patterns resulted being feature and game invariant, heterogeneous across subjects, but stable within subjects. Using a cluster analysis, we find correlations between eye-movements and choices; however, applying the Cognitive Hierarchy model to our data, we show that only some of the subjects present both information search patterns and choices compatible with a specific cognitive level. We also find a series of correlations between strategic behavior and individual characteristics like risk attitude, short-term memory capacity, and mathematical and logical abilities.

Keywords One-shot games - Eye-tracking - Focal points - Individual behavior * Bounded rationality · Feature-based choice

JEL Classification $\quad$ C72 $\cdot$ C91 $\cdot$ D01 $\cdot$ D83

\section{Introduction}

Most theories of behavior in games are based on the two fundamental assumptions of strategic thinking and optimization (Camerer et al. 2004): the former meaning that players develop beliefs about the likely behavior of other players, the latter implying that subjects best respond to these beliefs. Models that incorporate bounded rationality (however defined) allow beliefs and choices to be both heterogeneous and out of equilibrium, particularly before any learning process takes place: i.e. in games that are played only once or in initial behavior in repeated games. For example, models of limited cognition (Stahl and Wilson 1994, 1995; Nagel 1995; Ho et al. 1998; Costa-Gomes et al. 2001; Bosch-Domènech et al. 2002; Crawford 2003; Camerer et al. 2004; Costa-Gomes and Crawford 2006; Crawford and Iriberri 2007a, b) typically assume that players vary in their ability to perform iterated reasoning, and believe that other players are able to perform less steps than themselves (Camerer et al. 2004). However, players are still assumed to form a correct mental representation of the strategic situation at hand, and to apply forms of strategic reasoning that allow them to form a model of the other player(s), however imperfect and incorrect it may be.

However, isolated but important recent results seem to question the validity of these fundamental assumptions. Costa-Gomes and Weizsäcker (2008) show that choices are, on average, inconsistent with beliefs and subjects fail to best respond to their own stated beliefs in roughly half the games being tested. Devetag and Warglien (2008) show that subjects' mental models are systematically (and mistakenly) simplified, so as to reduce the game payoff structure from a mixed motive to a pure motive one. In a series of dominance solvable guessing games, Rydval et al. (2009) show that nearly two thirds of experimental subjects show reasoning inconsistent with dominance, although a quarter of them actually do choose dominant strategies. Using the eye-tracking, Arieli et al. (2011) show that 
subjects facing two lotteries often compare prizes and probabilities separately, rather than extensively analyzing the whole structure of each lottery, as suggested by expected utility theory. In Weizsäcker (2003), subjects behave as if they underestimate their opponents' rationality or ignore other players' choices when making their own decisions. Finally, it has been shown experimentally that some subjects focus their attention unevenly across the information at their disposal (Hristova and Grinberg 2005), while others collect information thoroughly (Brocas et al. 2014).

The evidence cited above points at two strictly interrelated phenomena; first, some players seem to ignore other players' motivations and incentives, possibly to simplify a choice problem that is perceived as cognitively demanding. Second, players apply decision rules that do not involve orthodox strategic reasoning and that are not based on a mental model that corresponds to the true game being played.

We hypothesize that in one-shot games, subjects best respond to a simplification of the original decision problem, obtained either by ignoring the other players motivations or by taking them into account only for a subset of all possible game outcomes. Di Guida and Devetag (2013) show that it is possible to induce systematic and predictable changes in players' behavior by manipulating a game set of descriptive features (i.e., features that can be changed without altering the game equilibrium properties). They suggest that descriptive features provide attractive solutions to subjects using boundedly rational decision rules. Only when these features are removed, subjects exert more effort into thinking strategically, and in some cases, choose Nash equilibrium strategies.

A recent approach to the study of gaming behavior aimed to shed light on players' strategic reasoning includes the use of process data. The analysis of information search patterns, together with a classical analysis of subjects' actual choices, allows investigating the subconscious mechanisms at the basis of strategizing. Techniques like mouse-tracking (Costa-Gomes et al. 2001; Johnson et al. 2002; Brocas et al. 2014), eye-tracking (Knoepfle et al. 2009; Wang et al. 2010; Arieli et al. 2011; Chen et al. 2013 mimeo; Fiedler et al. 2013), and fMRI (Bhatt and Camerer 2005) allow researchers to catch a glimpse of the cognitive mechanisms driving human strategic behavior while keeping them subconscious, and therefore avoiding the noise produced in the phase of "elicitation" (i.e. when subjects are asked to explain verbally why did they act in a particular way).

In this paper, we analyze subjects' eye movements (or "lookup patterns") when playing the games presented in Di Guida and Devetag (2013) to test whether the information search patterns subjects exhibit are more compatible with "boundedly rational decision rules" or with the "best responding to beliefs" hypothesis. Our data substantially confirm Di Guida and Devetag (2013) results. Analysis of lookup patterns shows that subjects perform partial or non-strategic analyses of the game, often ignoring the payoffs of the opponent. We also find a correlation between choices and lookup patterns, but interestingly, even though choices are affected by features, lookup patterns are neither affected by features nor by the game equilibrium structure; they appear to be heterogeneous across subjects, but largely invariant within subjects. 
Altogether, our findings strongly support the hypothesis that subjects in one-shot games in normal form apply boundedly rational decision rules that are based on a simplified model of the true situation.

\section{Games, experimental design, and behavioral predictions}

\subsection{The games}

As we are interested in initial behavior only, we implement a random rematching scheme with no feedback (as in Costa-Gomes et al. 2001), in order to minimize learning and "repeated game" effects. The payoff matrices used in the experiment are listed in Table 1.

The basic games (see Di Guida and Devetag 2013) are: a game with a strictly dominant strategy for the column player (henceforth, DomCol); a game without pure strategy Nash Equilibria (noNe), a game with a single pure strategy Nash Equilibrium but not solvable through iterated elimination of dominated strategies (UniqNe), a modified Prisoners' Dilemma (PD), and a Weak Link coordination game (WL).

As in Di Guida and Devetag (2013), we are interested in the effects produced by two descriptive features: the variance of the strategy giving the highest average payoff to the player whose behavior we intend to observe (henceforth HA), and the presence of an attractor (henceforth A). Manipulating its variance makes HA a safe and moderately appealing strategy when the variance is low and a riskier but tempting one when the variance is high. An attractor is any cell containing Paretoefficient and symmetric payoffs, located at the center of the matrix. ${ }^{1}$ Except in the Weak Link game, our attractors are not equilibria. To identify both features' separate and joint effects, we created a matrix for every possible combination of features. Six matrices were therefore created for each base game, for a total of 30 matrices.

To facilitate our exposition, we refer to each matrix by the acronym identifying the game type, and by two acronyms identifying its features: "A" means a matrix with an attractor, "NA" a matrix without attractor, and "Low", "Medium", and "High" the three levels of variance of the strategy with the highest average payoff. Since due to matrices' construction constraints we are only interested in row players behavior, all descriptions of features and matrices deal with the row player's perspective, unless otherwise specified. Given the structure of the matrices, we assume that agents choosing the row containing the attractor do so because of the attractor itself. Therefore, the row containing the attractor is labeled as "A strategy". Same holds for the row giving the highest average payoff, labeled as "HA strategy" (see Di Guida and Devetag 2013 for further details of the experimental design).

\footnotetext{
${ }^{1}$ In the Weak Link game all symmetric cells were positioned along the main diagonal from the highest to the lowest payoff.
} 


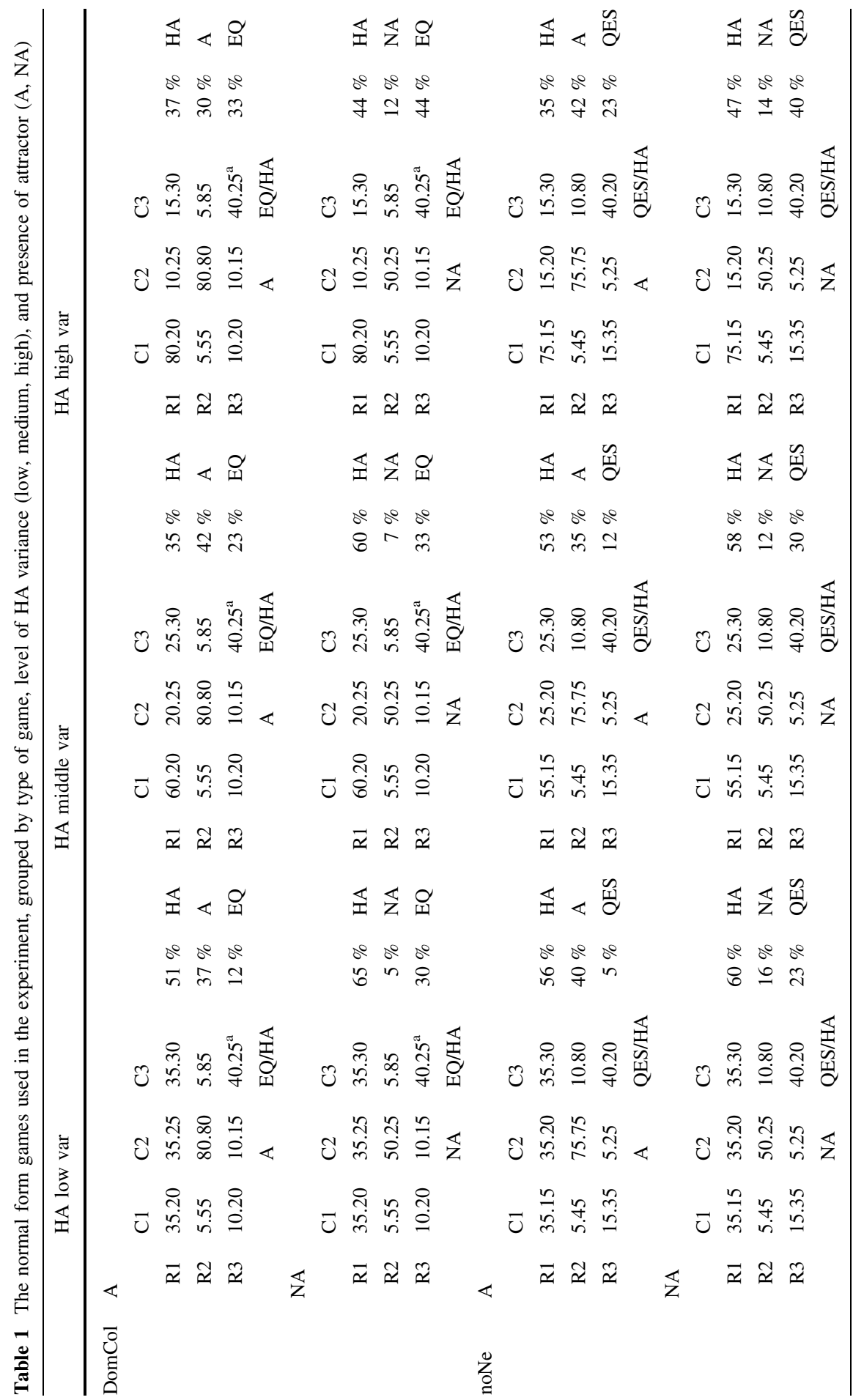




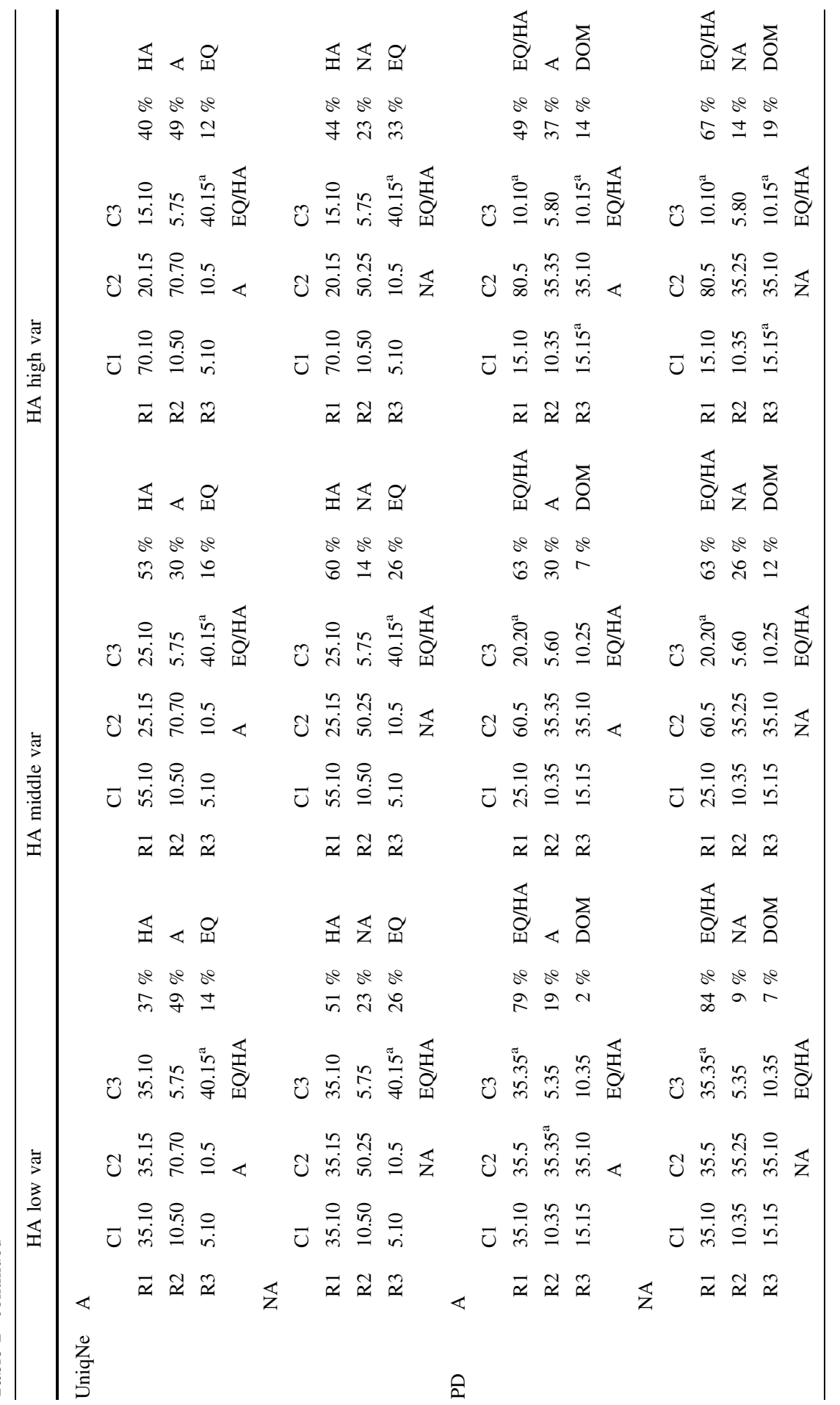




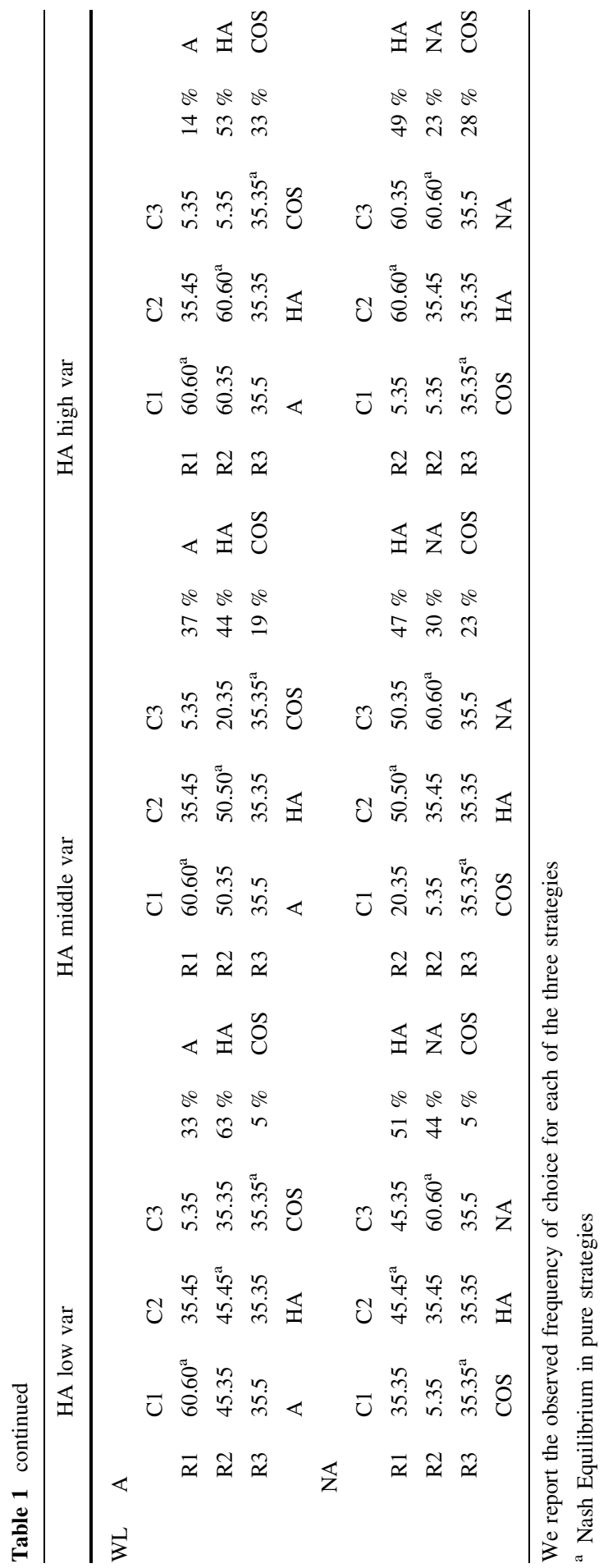


All versions of each game are created by modifying cells' content as little as possible and by maintaining unaltered the pure strategy Nash Equilibria. In a few cases, these changes added new Nash equilibria in mixed strategies. In extreme cases, two matrices differed by a single cell. Except in one matrix (WL A Low), the average payoff of the HA strategy remained invariant: only its distribution was modified as to change the value of payoff variance. In DomCol, noNe, and UniqNe, matrices without attractor are obtained by breaking the symmetry of payoffs and by substantially reducing payoffs' magnitude. For the PD we are obliged by the game structure to eliminate the attractor by breaking payoff symmetry only, without a significant reduction in payoff size. In the Weak Link, given that the attractor is the payoff-dominant equilibrium, we simply move the corresponding cell out of the main diagonal.

We keep our strategies of interest separate whenever possible. To avoid spurious effects due to the position of the strategies in the matrix, we always keep the position of every strategy fixed in the different versions of the same game, the only exception being the WL game. ${ }^{2}$

\subsection{Experimental design and implementation}

The experiment was conducted at the EPL lab (Experimental Psychology Laboratory) of the University of Trento. As we are only interested in the row players' behavior, we collected eye-tracking data for row players only, and matched each row player with a column player drawn at random from the pool of subjects participating in the experiment in Di Guida and Devetag (2013). The pairings differed across games. A total of 43 subjects were eye-tracked, all playing as row player. Before the experiment started, a printed copy of the instructions was given to the subject and read aloud by the experimenter. Control questions were administered to assure that the mechanism of the experiment was understood. ${ }^{3}$

Subjects were explicitly told that they would play in the role of row player, and that their choices would be matched with those of other subjects that had played before. It was specified that the payment would be calculated based on the outcomes of 3 randomly selected games. The mechanism of random selection was made explicit.

After the eye-tracking machinery was calibrated, subjects played four practice games. The order in which the 30 matrices were displayed was random and differed across subjects. Once the experiment was concluded, subjects had to complete a series of questionnaires aimed at measuring cognitive abilities, personality traits, and degree of risk aversion. The experiment lasted on average less than $1 \mathrm{~h}$, and average payment was 9.20 Euros (the average payment was calibrated according to the EPL lab guidelines). In each round, subjects had to select their preferred strategy

\footnotetext{
${ }^{2}$ Results in Di Guida and Devetag (2013) suggest that the position of a strategy does not affect its frequency.

${ }^{3}$ Detailed description of the experimental procedure and of the machinery calibration phase, translated copy of the instructions, and control questions are reported in Appendices A and B in supplementary materials.
} 
by pressing the keys " 1 ", " 2 ", or " 3 ", on the keyboard. No feedback was given until the end of the experiment.

\subsection{Eye-tracking data}

At each round, subjects are presented with a $3 \times 3$ payoff matrix. For each matrix, 18 areas of interest (AOIs) are defined, one for each of the 18 payoffs (Fig. 1). Each cell contains two areas of interest, centered on the row and column players' payoff. The AOIs of the row player are numbered from 1 to 9 , whereas those of the column player from 10 to 18 . AOIs do not overlap, nor cover the matrix area entirely, but only half of it approximately. In this way, AOIs include only eye-movements whose interpretation is not ambiguous. Although a large part of the matrix is not included in any AOIs, the majority of fixations observed (90\%) fell inside the AOIs.

For each subject and round, we record four types of variables. The first two are how many times (fixation count) and for how long (fixation time) a subject fixes a point inside (but also outside) an AOI. Since these two variables are usually strongly correlated, we will mostly refer to the first variable (fixation count or simply fixation). The third and fourth variables are the number and type of transitions, i.e. the eye-movements from one AOI to the next.

Considering all possible pairs of AOIs and assuming that each pair can be connected by two transitions (one for each direction), the number of transitions that

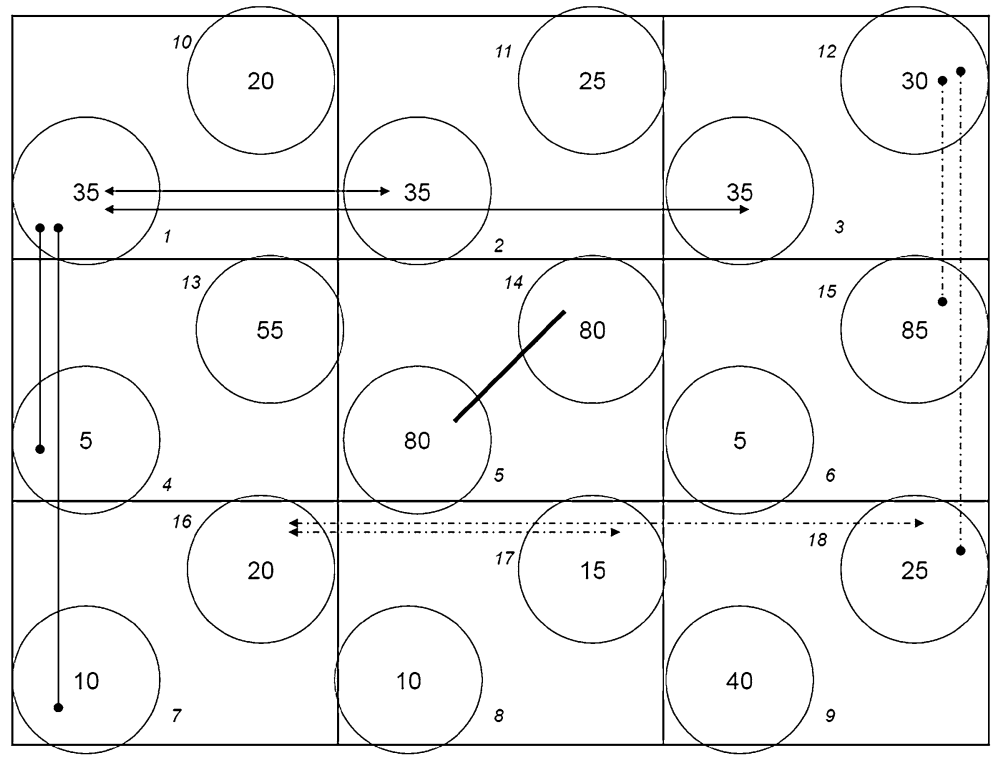

Fig. 1 Summary of the AOIs and transitions of interest; the numbers in italic represent the labels of the different Areas Of Interest (AOI), from 1 to 18. Transitions are represented as follows: Row Player by row (RPr): thin continuous line with arrows; Column Player by row (CPr): dashed line with arrows; Row Player by column (RPc): thin continuous line with circles; Column Player by column (CPc): dashed line with circles; Infracell (INF): thick continuous line 
could be potentially observed equals 324 , including transitions within the same AOI. However, only a subset of these is informative for our purposes. We consider the following five types of transitions-where AOI R corresponds to the AOIs of row players' payoffs and AOI C to those of column players' payoffs (see Fig. 1): Row Player by row (RPr), i.e. eye-movements from one AOI R to another AOI R lying in the same row; Column Player by row (CPr), i.e. eye-movements from one AOI $\mathrm{C}$ to another AOI C lying in the same row; Row Player by column (RPc), i.e. eye-movements from one AOI R to another AOI R lying in the same column; Column Player by column $(\mathrm{CPc})$, i.e. eye-movements from one AOI $\mathrm{C}$ to another AOI C lying in the same column; Payoffs infracell (INF), i.e. eye-movements from an $\mathrm{AOI} \mathrm{R}$ to an $\mathrm{AOI} \mathrm{C}$ or vice versa lying within the same cell. Transitions that remain within the same AOI are excluded.

According to our hypotheses, transitions can be interpreted as information search patterns, and are closely related to the decision rule adopted. Therefore, the analysis of transitions can provide insight about the type of rules used by the decision makers. For example, exploring the matrix exclusively or prevailingly through RPr transitions (Row Player by row) indicates a subject ignoring other players' choices. In a case like this, the decision maker may be calculating the average expected value of all strategies available in order to pick the one with the highest value, a process that requires summing up (and therefore observing) payoffs by row. RPc transitions (Row Player by column) are instead compatible with the detection of simple dominance, while CPr (Column Player by row) with the detection of dominant strategies for the column player, i.e., with performing one step of iterated dominance. CPc transitions (Column Player by column) might be used if looking for the strategy giving the column player the highest average payoff [behavior consistent with level-2 players, as suggested by Costa-Gomes et al. (2001), and by Bhatt and Camerer (2005)]. Finally, INF is compatible with a choice process based on the analysis of matrix cells, induced either by the presence of salient outcomes such as focal points or attractors, or by decision rules that focus on payoffs sums [like the "Altruistic" type, see Stahl and Wilson (1994, 1995), Costa-Gomes et al. (2001)], or payoffs differences [fairness, inequality aversion, competitive preferences,... see Rabin (1993); Fehr and Schmidt (1999); Bolton and Ockenfels (2000)].

\subsection{Behavioral hypotheses}

We formulate the following research hypotheses: first (Hypothesis 1: "featureschoices relation") we assume that players are influenced by the presence of "intuitive" and "easy" solutions; therefore, strategy HA when its variance is low, together with strategy A (leading to an attractive outcome) will be chosen more often than the equilibrium strategy, with the share of HA decreasing as its variance increases. Only when these features are absent, more players will switch to the equilibrium strategy.

Second (Hypothesis 2: "features-lookup patterns relation"), we assume that subjects on average perform partial or non-strategic analyses, paying attention only to specific subsets of the matrix elements. The attention depends both on game type and on feature composition, besides presumably varying across players. For 
example, we expect the "attractor" cell to attract more attention than the remaining cells, ceteris paribus.

Finally (Hypothesis 3: "lookup patterns-choices relation"), we assume a correlation between choices and lookup patterns. In particular, players who select HA tend to focus on their own payoffs (ignoring the opponents' payoffs) and are more prone to analyze the matrix by row; players who select A are more prone to
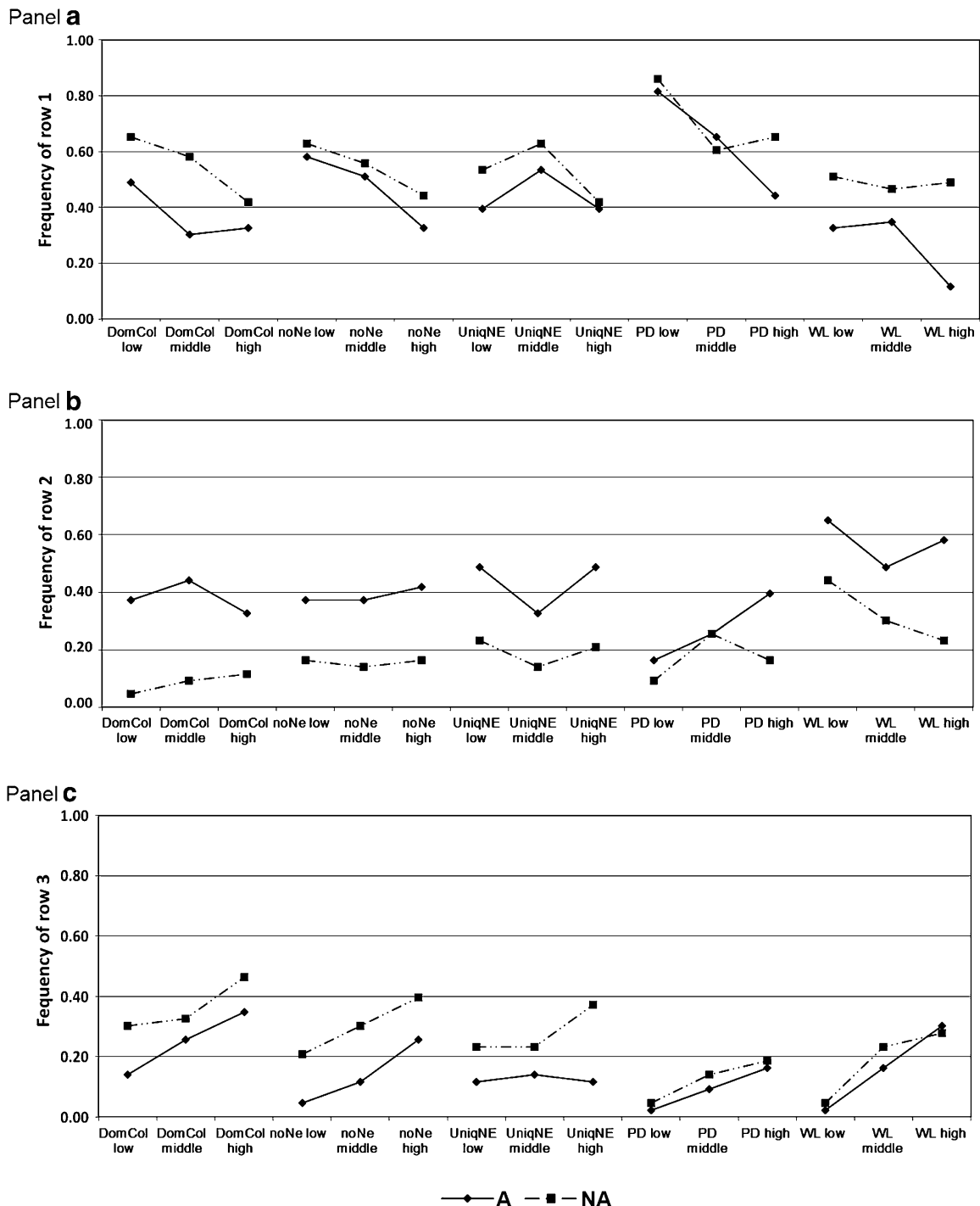

Fig. 2 a Observed frequencies of row 1 choices. b Observed frequencies of row 2 choices. c Observed frequencies of row 3 choices. Frequencies in matrices with attractor are marked with a continuous line, those in matrices without attractor with a dashed line 
analyze the game by cell (i.e., they present more infracell saccades) and pay relatively more attention to the attractor cell; players who select EQ perform on average a more complete game analysis (Costa-Gomes et al. 2001).

Hypothesis 2 states that subjects' use of decision rules is based on selective information processing, that is, a simplified model of the "true" situation. Hypothesis 3 states that specific choices are the result of specific decision rules, which are based on the aforementioned selective information processing. Both hypotheses are important to discriminate between explanations of behavior based on "best-reply-to-beliefs" and those based on the use of decisional shortcuts that rely on mental simplifications of the real decision problem.

\section{Results}

\subsection{Behavioral data}

Before moving to the lookup pattern analysis, we present an overview of choice data. Three eye-tracked observations had to be discarded because of low calibration quality. Therefore the subject pool is composed of 43 subjects in the aggregate analysis and 40 subjects in the lookup pattern analysis. A data overview is provided in Fig. 2. The difference in choice distributions between matrices with and without attractor is evident, as well as the effect due to the increase in the variance of strategy HA. A comparison between choice distributions in the A Low and in the NA High version of each game by a Bhapkar test reveals that differences are always significant at the $5 \%$ level, indicating that the presence or absence of features affects choices. In all games except the Weak Link, ${ }^{4}$ the frequency of the attractor strategy is higher in matrices with an attractor than in those without it. According to a binomial test, in the games DomCol, noNe, and UniqNe, the difference in choice shares is always significant with $\mathrm{p} \leq .05$ (except in UniqNe Middle where $\mathrm{p}=.07$ ). Also, according to a paired $t$ test, the average frequency of HA in games where variance is low is significantly higher than that in games where variance is high $(\mathrm{p}=.001)$, confirming that an increase in variance reduces the appeal of the HA strategy.

Overall, our Hypothesis 1 is confirmed, and the results in Di Guida and Devetag (2013) successfully replicated. Our descriptive features create "easy" choices: a safe and attractive strategy, and a strategy leading to a very attractive outcome for both players. The attractor in our game matrices can be labeled as a behavioral "focal point".

\footnotetext{
${ }^{4}$ In the WL NA matrices, the cell containing the attractor is not modified, but rows and columns are shuffled to move the attractor to a less "evident" position. As already shown in Di Guida and Devetag (2013), subjects' behavior indicates that this is not sufficient to reduce the cell attractiveness.
} 


\subsection{Overview of fixations}

We only consider fixations longer than $100 \mathrm{~ms}$, which have been proved a sufficient threshold to discriminate between fixations and other ocular activities (Manor and Gordon 2003).

Figure 3 shows the number of fixations by game type and by features combination. It is noteworthy that, moving from left to right, i.e., shifting from matrices with attractor to matrices without, and from low to high levels of variance of HA, the total number of fixations increases, confirming our hypothesis that the absence of attractive features makes a game harder to process. Notwithstanding this general tendency, however, the distribution of fixations across games appears markedly different. Some games (DomCol, noNe, and PD) are particularly sensitive to changes in descriptive features, as shown by their fixations increasing by $50 \%$ or more from A low to NA high games. UniqNe seems less feature-sensitive since only a slight increase in the number of fixations is observed, while the fixations of WL are almost constant across different versions. Overall attention was very unevenly distributed across the different elements of the game matrix. Fixations devoted to AOIs from 1 to 9 (comprising a subject's own payoffs) amount to 26,118, against the 20,554 fixations dedicated to AOIs from 10 to 18 . At the individual level, the two distributions are significantly different by a Wilcoxon signed rank test $(\mathrm{p}=.039)$. Hence, on average, subjects devote disproportionately more attention to their own payoffs compared to their opponents' payoffs, in line with what suggested by choice data from previous experiments (Costa-Gomes et al. 2001; Wang et al. 2010).

Figure $4 \mathrm{a}$ reports the absolute and relative frequencies of fixations in the matrices with (A) and without (NA) attractor, by cell. It is noteworthy that fixations in matrices with attractor are always less, in absolute terms, than those in matrices without attractor, except, as predicted, for the attractor cell. The two distributions, however, look almost identical (again with the exception of the attractor), suggesting that relative attention was invariant. Figure $4 \mathrm{~b}$ shows the absolute and relative frequency of fixations for each of the three variance levels of the HA strategy by cell. The graph shows that each cell is always observed less frequently in matrices with HA low variance than in those with medium and high variance. Distributions are again invariant, suggesting that increasing HA increases the amount of overall gazing time but does not per se change each cell relative importance. As expected, the attractor is the most looked at cell.

\subsection{Overview of transitions}

Figure $4 \mathrm{c}$ and $\mathrm{d}$ report the absolute and relative frequency of transitions by type, distinguishing between matrices with and without attractor (Fig. 4c), and between different levels of HA variance (Fig. 4d). The figures show that the most frequent transitions are RPr (Row Player by row) and INF (Payoffs infracell). The third most frequent category is that of $\mathrm{CPc}$ transitions. The observation suggests that subjects tend to compare strategies according to their average payoff ( $\mathrm{RPr}$ and $\mathrm{CPc}$ ), rather than by looking for dominance relation (RPc and $\mathrm{CPr}$ ). An equally frequent 


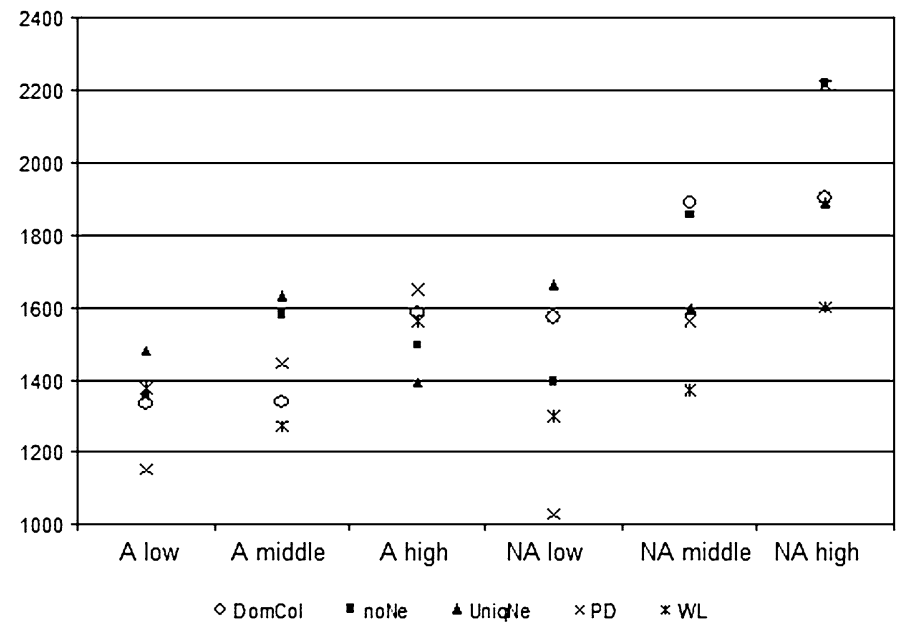

Fig. 3 Total fixations divided by game, by presence of attractor (A/NA), and by HA level

transition entails comparing payoffs within the same cell. Absolute frequencies of transitions are higher for matrices without attractors, and they increase as HA variance increases. Nonetheless, their relative frequency seems relatively unaffected by the presence or absence of features.

Figure $4 \mathrm{e}$ shows how transitions are distributed across different games and payoff matrices. As the graph shows, there is a clear and stable prevalence of RPr and INF over all typologies of transitions in each of the 30 games, despite substantial variations in absolute levels. Hence, the most frequently observed information processing patterns look roughly similar across all games.

\subsection{Features, choices, and lookup patterns}

This analysis aims to verify whether a correlation may be found between subjects' choices and their lookup patterns. In the experiment, a total of 40 subjects played 30 games each, for a total of 1200 choices. Of these, $40 \%$ were HA choices, $17 \%$ A, $15 \% \mathrm{EQ}$, and $13 \% \mathrm{EQ} / \mathrm{HA}^{5}$ Table 2 shows the correlation results. Shaded coefficients are those that resulted statistically significant at the 5 per cent level according to a Spearman correlation test.

Several considerations can be drawn by looking at correlation data: HA choices are positively and significantly correlated with $\mathrm{RPr}$ transitions $(\mathrm{r}=.47$, eyemovements that connect a player's own payoffs by row) and are negatively and significantly correlated with all transitions involving the opponents' payoffs, including INF transitions $(\mathrm{r}=-.46)$. Moreover, HA choices are also negatively and significantly correlated with all AOIs from 10 to 18, i.e., all AOIs related to the opponent's payoffs. This evidence strongly confirms our hypothesis that players who choose HA do so by employing a decision rule that prescribes to pick the

\footnotetext{
5 The others were: 9 per cent NA, 4 per cent COS, and 2 per cent DOM, see Table 1.
} 

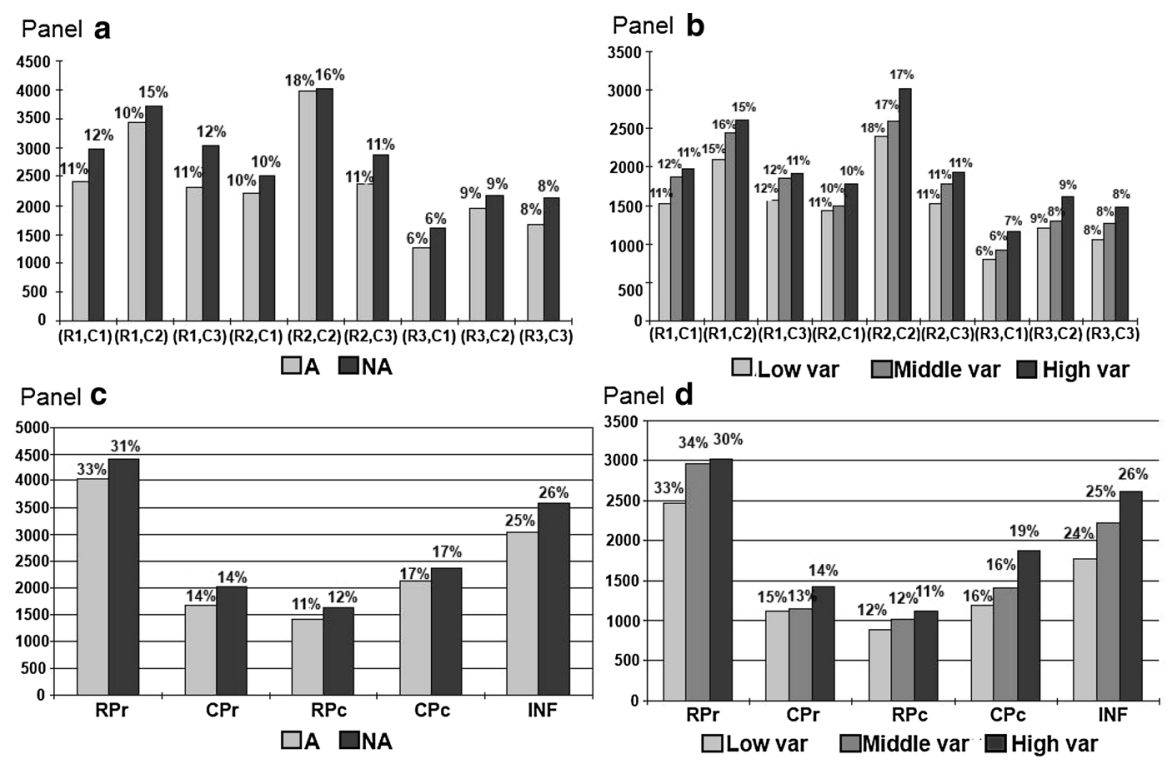

Panel e

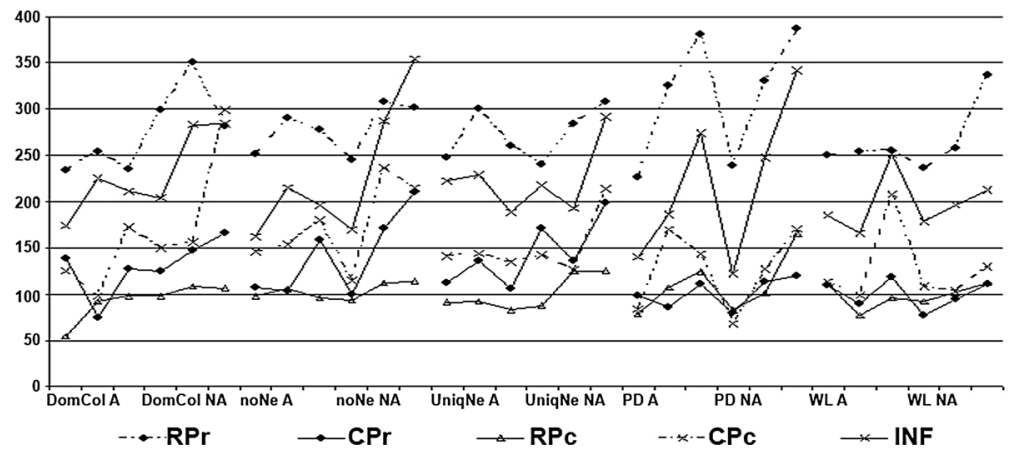

Fig. 4 a Absolute and relative frequency of fixations divided by cell, in matrices with attractor (A) and without attractor (NA). b Absolute and relative frequency of fixations divided by cell, in matrices with different variances of HA. c Absolute and relative frequency of transitions, in matrices with attractor (A) and without attractor (NA). d Absolute and relative frequency of transitions, in matrices with different variances of HA. e Absolute frequency of transitions, by game

strategy with the highest expected value ignoring the other players' motivations, and implicitly treating the strategic decision problem as an individual decision making problem.

A choices (choices of strategies leading to the attractor), as predicted, are positively and significantly correlated with INF transitions $(\mathrm{r}=.40)$, and with transitions connecting column players' payoffs by row $(r=.46)$. A choices are also positively and significantly correlated with the attractor cell, (R2, C2) and with a subset of the opponent's AOIs, namely AOIs 10 and 11, and 13 and 14, corresponding to payoffs in the first and second row of the matrix. As assumed, 
Table 2 Correlation between choices (only the main categories were considered), transitions, and number of fixations (by cell and by AOI)

\begin{tabular}{|c|c|c|c|c|c|c|c|c|}
\hline & EQ choices & A choices & HA choices & $\mathrm{RPr}$ & $\mathrm{CPr}$ & $\mathrm{RPc}$ & $\mathrm{CPC}$ & INF \\
\hline EQ choices & & 0.03 & -0.45 & -0.18 & 0.35 & 0.34 & 0.69 & 0.46 \\
\hline A choices & 0.03 & & -0.64 & -0.15 & 0.46 & -0.09 & 0.18 & 0.40 \\
\hline HA choices & -0.45 & -0.64 & & 0.47 & -0.56 & -0.08 & -0.53 & -0.46 \\
\hline $\mathrm{RPr}$ & -0.18 & -0.15 & 0.47 & & 0.21 & 0.54 & 0.08 & 0.07 \\
\hline $\mathrm{CPr}$ & 0.35 & 0.46 & -0.56 & 0.21 & & 0.43 & 0.73 & 0.79 \\
\hline $\mathrm{RPC}$ & 0.34 & -0.09 & -0.08 & 0.54 & 0.43 & . & 0.58 & 0.24 \\
\hline $\mathrm{CPC}$ & 0.69 & 0.18 & -0.53 & 0.08 & 0.73 & 0.58 & & 0.62 \\
\hline INF & 0.46 & 0.40 & -0.46 & 0.07 & 0.79 & 0.24 & 0.62 & \\
\hline (R1,C1) Cell & 0.23 & 0.27 & -0.17 & 0.55 & 0.74 & 0.46 & 0.54 & 0.76 \\
\hline (R1,C2) Cell & 0.18 & 0.16 & -0.11 & 0.70 & 0.73 & 0.60 & 0.57 & 0.64 \\
\hline (R1,C3) Cell & 0.54 & 0.07 & -0.24 & 0.40 & 0.75 & 0.59 & 0.81 & 0.78 \\
\hline (R2, C1) Cell & 0.37 & 0.29 & -0.34 & 0.47 & 0.84 & 0.52 & 0.68 & 0.79 \\
\hline (R2,C2) Cell & 0.25 & 0.34 & -0.30 & 0.53 & 0.78 & 0.63 & 0.63 & 0.75 \\
\hline (R2,C3) Cell & 0.59 & 0.27 & -0.44 & 0.29 & 0.84 & 0.60 & 0.84 & 0.84 \\
\hline (R3,C1) Cell & 0.36 & 0.00 & -0.02 & 0.62 & 0.65 & 0.59 & 0.57 & 0.65 \\
\hline (R3,C2) Cell & 0.22 & 0.02 & 0.09 & 0.71 & 0.61 & 0.59 & 0.46 & 0.56 \\
\hline (R3,C3) Cell & 0.70 & 0.07 & -0.32 & 0.25 & 0.71 & 0.56 & 0.82 & 0.78 \\
\hline AOI 1 & 0.01 & 0.13 & 0.14 & 0.77 & 0.48 & 0.45 & 0.28 & 0.50 \\
\hline $\mathrm{AOI} 2$ & -0.07 & 0.01 & 0.22 & 0.87 & 0.44 & 0.57 & 0.30 & 0.37 \\
\hline $\mathrm{AOI} 3$ & 0.35 & -0.02 & -0.01 & 0.62 & 0.61 & 0.61 & 0.64 & 0.65 \\
\hline $\mathrm{AOI} 4$ & 0.03 & 0.11 & 0.10 & 0.71 & 0.49 & 0.39 & 0.26 & 0.54 \\
\hline AOI 5 & -0.08 & 0.21 & 0.09 & 0.79 & 0.48 & 0.55 & 0.23 & 0.43 \\
\hline AOI 6 & 0.47 & 0.17 & -0.19 & 0.53 & 0.69 & 0.65 & 0.65 & 0.74 \\
\hline $\mathrm{AOI} 7$ & 0.07 & -0.14 & 0.36 & 0.78 & 0.33 & 0.46 & 0.17 & 0.36 \\
\hline AOI 8 & -0.07 & -0.07 & 0.41 & 0.89 & 0.29 & 0.56 & 0.12 & 0.22 \\
\hline AOI 9 & 0.48 & 0.02 & -0.05 & 0.51 & 0.54 & 0.62 & 0.57 & 0.58 \\
\hline $\mathrm{AOI} 10$ & 0.48 & 0.36 & -0.46 & 0.21 & 0.87 & 0.38 & 0.73 & 0.92 \\
\hline AOI 11 & 0.49 & 0.31 & -0.47 & 0.25 & 0.92 & 0.45 & 0.82 & 0.87 \\
\hline $\mathrm{AOI} 12$ & 0.66 & 0.20 & -0.49 & 0.08 & 0.82 & 0.46 & 0.90 & 0.83 \\
\hline AOI 13 & 0.49 & 0.33 & -0.47 & 0.26 & 0.90 & 0.49 & 0.80 & 0.85 \\
\hline AOI 14 & 0.49 & 0.37 & -0.54 & 0.21 & 0.89 & 0.53 & 0.84 & 0.85 \\
\hline $\mathrm{AOI} 15$ & 0.62 & 0.26 & -0.54 & 0.12 & 0.83 & 0.51 & 0.89 & 0.83 \\
\hline AOI 16 & 0.59 & 0.19 & -0.39 & 0.21 & 0.86 & 0.42 & 0.80 & 0.86 \\
\hline AOI 17 & 0.56 & 0.24 & -0.41 & 0.24 & 0.87 & 0.44 & 0.81 & 0.85 \\
\hline AOI 18 & 0.69 & 0.18 & -0.50 & 0.05 & 0.80 & 0.44 & 0.90 & 0.83 \\
\hline
\end{tabular}

Shaded coefficients are statistically significant at the $5 \%$ level

players who pick strategy A do take into account their opponent's payoffs, but focus their attention on a subset of possible game outcomes.

Choices of the equilibrium strategy (EQ) are positively and significantly correlated with the following transitions: $\operatorname{CPr}(r=.35), \operatorname{RPc}(r=.34), \operatorname{CPc}$ $(\mathrm{r}=.69)$, and INF $(\mathrm{r}=.46)$. They are positively and significantly correlated with AOIs 3, 6, and 9, i.e., with the player's own payoffs in the third column of the matrix. Moreover, EQ choices are positively and significantly correlated with all AOIs from 10 to 18 , that is, AOIs corresponding to all opponent's payoffs. In addition, the correlation with all cells belonging to the third column of the matrix (corresponding to the opponent's choice in equilibrium) is likewise positive and significant. This evidence strongly supports the idea that players who choose the equilibrium strategy are by far the most strategic in the standard game-theoretic meaning, processing the matrix according to eye-movements that suggest the search for dominance (RPc if looking for a dominant strategy for themselves, CPr if looking for a dominant strategy for the opponent) or for the strategy giving the opponent the highest average payoff (using CPc transitions and behaving as a level- 
2 player), paying attention to the opponents' payoffs for all possible game outcomes, and to cells of the matrix which other player types typically neglect (like the ones on the third column).

Correlations support hypothesis 3. Assuming that any information that is not looked at by the decision maker cannot enter the decision process, we find evidence that many players in one-shot games apply boundedly rational decision rules that simplify the decision problem either by ignoring the opponents' payoffs, or by considering them only for a subset of all possible outcomes. ${ }^{6}$

\subsection{Cluster analysis}

To further investigate to what extent lookup patterns are correlated with choices, we split our matrices into two equal-sized groups, use the first group to categorize subjects according to their lookup patterns, ${ }^{7}$ then test whether the patterns observed are correlated with the choices expressed in the second group of matrices. ${ }^{8} \mathrm{We}$ only use games DomCol, noNe, and UniqNe (as in these games the three features of interest are separate) and randomly divide the resulting 18 games into two groups of 9 games each.

We perform a cluster analysis using the normal distribution of the five transition types classified in Sect. 2.3 (RPr, RPc, CPr, CPc, INF). For this purpose, we used the mixture model presented in Brocas et al. (2014) and proposed by Fraley and Raftery (2002). Mixture models treat each cluster as a component probability distribution. A bayesian approach was then used to choose among different cluster numbers and statistical methods. As in Brocas et al. (2014), we considered a maximum of nine clusters and ten possible models, and we choose the combination that maximizes the Bayesian Information Criterion (BIC). With our data the BIC is maximized at -356 by a "diagonal model, varying volume and shape" yielding 3 clusters.

Figure 5a shows the normalized proportions of the different transitions (RPr, $\mathrm{RPc}, \mathrm{CPr}, \mathrm{CPc}$ INF) that subjects belonging to different clusters employ to analyze the games. Lookup patterns appear markedly different across clusters. In cluster 1 (10 subjects), agents focus entirely on their own payoffs, using mostly RPr transitions. Subjects like these-neglecting the opponent's payoffs-are expected to choose HA, since they did not acquire the information necessary to locate the equilibrium or the attractor. In cluster 2 (16 subjects), agents use mainly transitions connecting the other player's payoffs (especially by column). These subjects collect information more carefully than those belonging to cluster 1, and the use of CPc transitions suggests that they might be looking for the strategy giving the column player the highest average payoff [behavior consistent with level-2 players, as

\footnotetext{
${ }^{6}$ We also used Mediation Analysis to test whether lookup patterns are affected by features, and in turn affect choices. Causality between presence of features and choices is significant, but no significant relation is observed between features and lookup patterns..

${ }^{7}$ Matrices: DomCol_A_low, DomCol_NA_low, Domcol_NA_hig, noNe_A_middle, noNe_A_high, noNe_NA_middle, UniqNe_A_middle, UniqNe_NA_low, UniqNe_NA_middle.

${ }^{8}$ Matrices: DomCol_A_middle, DomCol_A_high, Domcol_NA_middle, noNe_A_low, noNe_NA_low, noNe_NA_high, UniqNe_A_low, UniqNe_A_high, UniqNe_NA_high.
} 


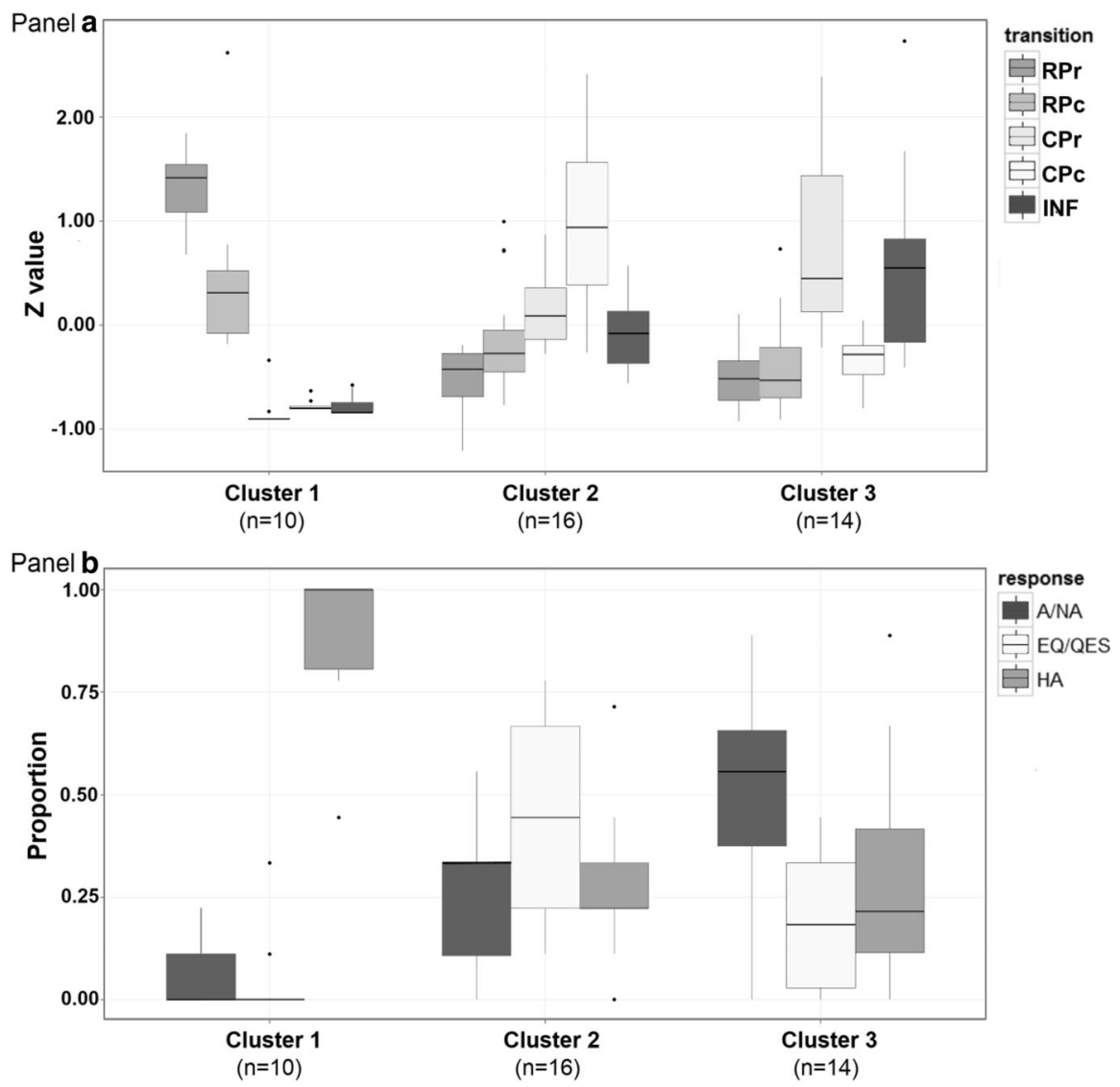

Fig. 5 a Boxplot summarizing the individual normalized proportion of different transitions, by cluster. b Boxplot summarizing the individual proportions of HA, A/NA, and EQ/QES choices, by cluster

suggested by Costa-Gomes et al. (2001), and by Bhatt and Camerer (2005)]. In the 18 games we are using for the cluster analysis, the best reply to a column player choosing HA is always EQ, and this is what we expect subjects belonging to cluster 2 to choose. Lastly, in cluster 3 (14 subjects) agents devote considerable attention to the other player's payoffs, but use many more infracell transitions than any other cluster. The large use of CPr transitions suggests that subjects might be looking for dominant strategies for the opponent, while the use of infracell transitions suggests that they might be also looking for cells resulting attractive for both players. These lookup patterns depict a row player carefully considering how to reply to the two most likely strategies the column player might adopt: whether choosing a dominant strategy or trying to coordinate. We expect these subjects to choose A whenever the attractor is present and EQ otherwise.

Figure $5 \mathrm{~b}$ reports the proportions of $\mathrm{HA}, \mathrm{A} / \mathrm{NA}$, and EQ/QES choices for each cluster. The figure confirms the relationship between transition types and choices: 
players in cluster 1 analyze their own payoffs by row and select HA almost exclusively ( $89 \%$ HA, $7 \%$ A/NA, and $4 \%$ EQ choices, on average). Players in cluster 2 use mostly CPc transitions and show the highest frequency of EQ choices, together with a minor proportion of HA and A/NA $(44,26$, and $29 \%$ on average, respectively). Cluster 3 shows the largest use of infracell transitions and the highest number of A/NA choices (49 \% on average), followed by HA and EQ choices (32 and $20 \%$ respectively). In cluster 3 , players mostly choose A when the attractor is present ( $65 \%$ of the times, in 5 out of 9 games), and switch to another strategy when the attractor is removed (30\% of NA choices in the remaining 4 games).

\subsection{The cognitive hierarchy model applied to our data}

Games like ours, built so that the use of different decision rules would lead to different choices, naturally suggests testing predictions of type-based or level-k models. Because of its tractability, its single parameter (corresponding to the average reasoning level of the subject sample), and of other specific characteristics, ${ }^{9}$ we decided to test the Cognitive Hierarchy model. In this model (Camerer et al. 2004) subjects are divided into different strategic categories according to their level of sophistication. Each subject assumes to be more sophisticated than the others, and chooses her strategy as the best response to a distribution of opponents (distributed according to a Poisson) ranging from level 0 to level $\mathrm{k}-1$, where $\mathrm{k}$ is the level of sophistication of the subject herself. Costa-Gomes et al. (2001), Bhatt and Camerer (2005), and Brocas et al. (2014) define the pattern of information acquisition employed by players exhibiting different levels of strategic thinking when playing normal form games. In line with these researches, we expect level-0 players to mostly ignore the information at their disposal and level-1 players to focus entirely on their own payoffs, using RPr transitions to calculate the expected value of each strategy or RPc to look for dominance relations. Level-2 players analyze thoroughly the payoffs of both players; more specifically, they are expected to use CPc and CPr transitions to draw considerations about the opponent's behavior, and RPc in order to choose their best response once the probable choice of the opponent has been singled out (as well as to look for a possible dominant strategy). For players of higher levels we expect a mixture of all the transitions connecting the payoffs of the same player; however, for no one we expect to observe infracell transitions.

We estimate $\tau$ on the same games used for creating our clusters and then compare the Cognitive Hierarchy predictions with the clusters we obtained. We estimated the parameters by choosing the value that minimizes the mean square deviation (MSD) between observed and estimated frequencies. The parameter value that best fits the observed choices in the nine games of interest is $\tau=0.34$, which implies that 28 of our subjects should behave as level-0 (71\%), 10 as level-1 (25\%), and 2 as level-2 (4\%). We also obtain 3 clusters, but our subjects' lookup patterns are only partially compatible with those predicted by the Cognitive Hierarchy model. Subjects in Cluster 1 are behaving as level-1 subjects, i.e. they focus almost entirely on their

\footnotetext{
9 As specified in Camerer et al. (2004), other level-k models make implausible predictions in some games and exhibit increasingly irrational expectations.
} 
own payoffs (in the first set of games on average $95 \%$ of the transitions are either RPr or RPc) and choose strategy HA almost exclusively (in the second set of games, on average, $89 \%$ of the choices corresponds to HA). Also, the number of subjects belonging to Cluster 1 is the same predicted by the Cognitive Hierarchy model for level-1 subjects.

Subjects in Cluster 2 behave in a way compatible with level- 2 subjects (i.e. they use mostly $\mathrm{CPc}$ and $\mathrm{CPr}$ transitions: on average $32 \%$ and $16 \%$ respectively), but for the large number of infracell transitions (19\%). Moreover, 16 of our subjects belong to Cluster 2, while only 2 were predicted by the model. Lastly, for $\tau=0.34$, A/NA choices should never be made by level-2 subjects other than by error, while we observe (on average) $26 \%$ of A/NA choices.

The most intriguing behavior is the one exhibited by subjects belonging to Cluster 3, where agents use every type of transition with equal frequency (34\% connecting their own payoffs, $30 \%$ connecting the opponent's payoffs, $36 \%$ infracell) and select every possible strategy, albeit with a strong preference for A/NA (49 \% on average). Such an extended use of every transition type and strategy might be compatible with level-3 (or higher) subjects. However, the Cognitive Hierarchy model does not allow for subjects of any level to use infracell transitions and in order to have that many subjects of level-3 or higher we should have almost no level-0 and level-1 subjects. Could subjects in Cluster 3 be "refined" level-0 subjects, who do not simply choose randomly all the time, but look for attractors and select them whenever present while switching to random when no attractors are available? We tend to exclude this possibility, given that subjects with such an unrefined strategy would be expected to devote less time and attention to the game structure than subjects of higher levels. On the contrary, subjects in Cluster 3 use only slightly less transitions in each game than subjects in Cluster 2 (on average 26.2 against 27.9) and much more than subjects in Cluster 1 (11).

This contrast between apparently unsophisticated choices (as suggested by the Cognitive Hierarchy parameter estimation) and sophisticated and stable information search patterns supports our hypothesis that subjects approach the game with a clear decision rule in mind and switch to a different one when the preferred one is not applicable, rather than modify their strategic sophistication across games. Our results partially challenge and partially support the level-k and type-based approaches. On the one hand, they challenge the models by showing that subjects do not consistently behave according to a specific cognitive level (or type). On the other hand, they support the models by showing that subjects behave consistently in games sharing the same features. Our findings, hence, suggest that a type-based approach is indeed appropriate, but that each type should allow subjects to apply different strategies to different game types (as identified by features). In other words, in order to use level-k or type-based models to predict behavior in certain games, the parameters should be estimated not on generic game classes, but on games sharing features.

\subsection{Cognitive and personality traits}

In this section we report the most relevant results of correlation tests (Spearman) among variables related to strategic behavior (choices and eye-movements) and a 
series of variables that should capture cognitive and personality traits likely to matter in the strategic task at hand (For a summary of complete results as well as a detailed explanation of the tests, see Appendix $\mathrm{C}$ in supplementary materials).

After the experiment, subjects were asked to complete a questionnaire containing: an immediate free recall working memory test (Unsworth and Engle 2007), a Wechsler Digit Span test for short memory (Wechsler 1987; Walsh and Betz 1990), the Cognitive Reflection Test (Frederick 2005), the Holt and Laury Risk Aversion test (Holt and Laury 2002), a test of Theory of Mind (Baron-Cohen 2004), and some cognitive and personality questionnaires (Rydval et al. 2009).

Several interesting findings emerge. First, risk aversion (as measured by the H\&L lottery test) is positively and significantly correlated with transitions of the RPr type $(\mathrm{r}=.37)$, suggesting that players who are more risk averse tend to process their own payoffs by row, a behavior compatible with the choice of HA. ${ }^{10}$ Risk aversion is also negatively correlated with equilibrium choices $(r=-.43)$. The Wechsler Digit Span test measures short term memory capacity (for details see Walsh and Betz 1990): we observe that scores in the digit span test (where a high score indicates high short term memory capacity) are positively correlated with the number of EQ choices $(r=.37)$, suggesting that subjects who pick equilibrium strategies are on average more capable of processing information. The score in the digit span test is also positively correlated with transitions that connect column players' payoffs $(\mathrm{CPr} r=.35$; $\mathrm{CPc} \mathrm{r}=.53$ ), and transitions that connect row players' payoffs by column (RPc $r=.54)$. Besides, there is a positive and significant correlation between individual score in the digit span test and all the AOIs that concern the other players' payoffs, as well as all the AOIs of the row player located in the third column (the column that in 18 out of 30 games corresponds to the equilibrium choice). Finally, the test is also positively correlated with a subject's strategic IQ, ${ }^{11}$ confirming the importance of short term memory capacity in strategic reasoning and strategic 'performance' $(r=.46)$. The strategic IQ is also positively correlated with EQ choices $(r=.80)$, while negatively correlated $(\mathrm{r}=-.35)$ with HA choices.

Of the various tests presented in Rydval et al. (2009), an interesting finding regards the Math Anxiety test (a small score indicates a relaxed feeling towards math), which is positively correlated with A choices $(r=.40)$ and negatively correlated with EQ choices $(\mathrm{r}=-.31, \mathrm{p}$-value $=.053)$. The Sensation Seeking test (where a small score indicates a risk seeking attitude) is negatively correlated with A choices $(\mathrm{r}=-.35)$.

The above findings support the conclusion that the ability to correctly incorporate the other players' incentives and motivations into ones own decision making process is strongly correlated with measures of individual capacity to process information as well as with some personality traits; in particular, short term memory

\footnotetext{
${ }^{10}$ The lack of a significant correlation between risk aversion and number of HA choices is most likely due to the fact that players, being risk averse, end up not selecting HA when its variance is high or medium. Hence, this finding strongly confirms the relevance of the risk factor in inducing a choice based on a strategy average payoff.

${ }^{11}$ Following Bhatt and Camerer (2005), we calculate each subject's 'strategic IQ' simply as his or her expected payoff.
} 
constraints seem to be able to explain a relevant part of the observed heterogeneity in game playing. Overall, these findings suggest that off-equilibrium choices in a variety of games may be a matter of bounded rationality rather than non-standard preferences or wild beliefs.

\section{Discussion and conclusion}

In this paper we extend the analysis started in Di Guida and Devetag (2013) by investigating the relationship between features, information search patterns (inferred from analysis of eye-movements), and choices. As previously shown (Costa-Gomes et al. 2001; Knoepfle et al., 2009; Wang et al. 2010; Chen et al. 2013 mimeo; Brocas et al. 2014; Stewart et al., 2015 mimeo), our subjects' information search patterns are highly correlated with their strategic behavior. However, and rather counterintuitively, while features have a direct influence on players' strategic behavior, they do not seem to affect information search patterns. Patterns appear to be relatively game and feature-invariant, stable within subjects, but largely variable across subjects.

More specifically, we show that subjects' choices in one-shot games are susceptible to the influence of equilibrium-irrelevant features in systematic and predictable ways. We posit that this effect can be adequately explained by assuming that players use decision rules that are based on a simplification of the decision problem, which may or may not involve neglecting the other player's incentives. We assume that the presence of an attractor and the presence of a strategy with an attractive risk-return profile offer easy and convenient "solutions" to the game being played. Only in the absence of such features may subjects engage in a more complete game analysis and employ more strategic decision criteria, including selecting the equilibrium strategy. Our hypotheses concern modal behavior only; hence, we expect heterogeneity in choices, which we assume to be correlated with heterogeneity in patterns of information analysis.

By studying subjects' eye movements, we observe that most subjects analyze the game only partially, paying disproportionately more attention to their own payoffs as opposed to the other player's payoffs (Costa-Gomes et al. 2001; Wang et al. 2010; Brocas et al. 2014), and to some of the matrix cells (e.g., the cell containing the attractor) as opposed to other cells (Hristova and Grinberg 2005). We also show that a non-negligible subset of subjects never look at the opponent's payoff, implicitly transforming the game into an individual decision making problem. Contrary to our expectations (but as observed also in Stewart et al. 2015 mimeo), our analyses of transitions reveal that lookup patterns are relatively game and feature-invariant.

As hypothesized, we find strong correlations between choices and lookup patterns. On average, the lookup patterns necessary for detecting dominance are the least observed (CRr and RPc), suggesting that looking for dominance relations is not at all an "obvious" and "intuitive" operation, as traditional game theory would suggest. A cluster analysis based on eye movements supports this observation, showing that subjects who focus on their own payoffs (some of which totally neglecting the opponent's payoffs) choose the strategy with the highest average 
payoff for themselves (acting as level-1 agents), whereas subjects who choose the strategy leading to the attractor the most are also those who use transitions that connect payoffs within cell the most. Finally, subjects who most often select the equilibrium strategy (which in many of our games coincides with a level-2 strategy), on average, perform a more complete game analysis, and in particular pay attention to the other player's payoffs by column, in line with a level-2 approach to the game.

Applying the Cognitive Hierarchy model (Camerer et al. 2004) to our data, we show that only some of the subjects present both information search patterns and choices compatible with a specific level of reasoning and that our feature-based approach gives a reasonable explanation for the large variance in parameter values observed. These findings, together with the results in Di Guida and Devetag (2013) on the application of the model in Costa-Gomes et al. (2001), suggest that in order to use level-k, and type-based models to predict subjects' behavior it is necessary to estimate their parameters on games that share features.

Finally, part of the heterogeneity observed can be explained by differences in risk attitudes and in short term memory capacity, in line with previous results on game playing (Devetag and Warglien 2008; Rydval et al. 2009).

It is important to stress that the decision rules driving our subjects' choices are presumably not the only ones at work in one-shot games. In fact, more than pinning down the specific choice criteria employed, our study aims to show that players may apply decision rules which (1) are based on an incomplete/imperfect model of the strategic situation at hand, and (2) are context-dependent; more specifically, they are sensitive to features of the game other than its equilibrium properties. Moreover, we believe heuristic-based behavior extends well beyond the games presented here and that deciding on the basis of a risk-return calculation is common in many games. Attractors, as defined here, can be present in many games: for example, both the payoff-dominant equilibrium in the stag hunt game and the mutual cooperation outcome in the Prisoner's Dilemma are attractors. Likewise, players may pick dominant strategies in dominant-solvable games not because they recognize the dominance relation (in fact, eye-movements compatible with the mental operation of checking dominance relations are rarely observed in our database), but because by definition these strategies have the highest average payoff.

Acknowledgments Financial supports from the "Italian Ministry of Education, University and Research" (MIUR, project PRIN 2008), from the "Fonds de la Recherche Fondamentale Collective" (research grant "Preference dynamics in adaptive networks", $\mathrm{n}^{\circ}$ 2.4614.12) and from the "Fondazione Cassa di Risparmio di Perugia" (grant "Ricerca di Base 2010") are gratefully acknowledged. We thank participants in the ESA 2011 North American meeting, the 6th Nordic Conference on Behavioral and Experimental Economics at Lund University, the Workshop on Rationality, Heuristics and Motivation in Decision Making at Scuola Normale Superiore in Pisa, and seminar participants at the University of Strasbourg and Luiss Guido Carli in Rome for useful comments and suggestions. The usual disclaimer applies.

\section{References}

Arieli, A., Ben-Ami, Y., \& Rubinstein, A. (2011). Fairness motivations and procedures of choice between lotteries as revealed through eye movements. American Economic Journal: Microeconomics, 3(4), $68-76$. 
Baron-Cohen, S. (2004). Questione di Cervello. La Differenza Essenziale tra Uomini e Donne. Mondadori.

Bhatt, M., \& Camerer, C. F. (2005). Self-referential thinking and equilibrium as states of mind in games: fMRI evidence. Games and Economic Behavior, 52(2), 424-459.

Bolton, G. E., \& Ockenfels, A. (2000). A theory of equity, reciprocity, and competition. American Economic Review, 90(1), 166-193.

Bosch-Domènech, A., Montalvo, J. G., Nagel, R., \& Satorra, A. (2002). One, two, (three), infinity,...: newspaper and lab beauty-contest experiments. American Economic Review, 92(5), 1687-1701.

Brocas, I., Carrillo, J. D., Wang, S. W., \& Camerer, C. F. (2014). Imperfect choice or imperfect attention? Understanding strategic thinking in private information games. The Review of Economic Studies, 81(3), 944-970.

Camerer, C. F., Ho, T.-H., \& Chong, J.-K. (2004). A cognitive hierarchy model of games. The Quarterly Journal of Economics, 119(3), 861-898.

Chen, C.-T., Huagn, C.-Y., \& Wang, J. T.-Y. (2013). A window of cognition: Eyetracking the reasoning process in spatial beauty contest games. http://citeseerx.ist.psu.edu/viewdoc/download?doi=10.1.1. 405.5663\&rep=rep1\&type=pdf. Accessed 24 Feb 2015.

Costa-Gomes, M., \& Crawford, V. P. (2006). cognition and behavior in two-person guessing games: An experimental study. American Economic Review, 96(5), 1737-1768.

Costa-Gomes, M., Crawford, V. P., \& Broseta, B. (2001). Cognition and behavior in normal-forma games: An experimental study. Econometrica, 69(5), 1193-1235.

Costa-Gomes, M., \& Weizsäcker, G. (2008). Stated beliefs and play in normal-form games. Review of Economic Studies, 75(3), 729-762.

Crawford, V. P. (2003). Lying for strategic advantage: rational and boundedly rational misrepresentation of intentions. American Economic Review, 93(1), 133-149.

Crawford, V. P., \& Iriberri, N. (2007a). Fatal attraction: salience, naiveté, and sophistication in experimental "hide-and-seek" games. American Economic Review, American Economic Association, 97(5), 1731-1750.

Crawford, V. P., \& Iriberri, N. (2007b). Level-k auctions: Can a non-equilibrium model of strategic thinking explain the Winner's curse and overbidding in private-value auctions? Econometrica, 75(6), 1721-7170.

Devetag, G., \& Warglien, M. (2008). Playing the wrong game: An experimental analysis of relational complexity and strategic misrepresentation. Games and Economic Behavior, 62(2), 364-382.

Di Guida, S., \& Devetag, G. (2013). Feature-based choice and similarity perception in normal-form games: An experimental study. Games, 4, 776-794.

Fehr, E., \& Schmidt, K. M. (1999). A theory of fairness, competition. And Cooperation. Quarterly Journal of Economics, 114(3), 817-868.

Fiedler, S., Glöckner, A., Nicklish, A., \& Dickert, S. (2013). Social value orientation and information search in social dilemmas: An eye-tracking analysis. Organizational Behavior and Human Decision Processes, 120(2), 272-284.

Fraley, C., \& Raftery, A. E. (2002). Model-based clustering, discriminant analysis, and density estimation. Journal of the American Statistical Association, 97(458), 611-631.

Frederick, S. (2005). Cognitive reflection and decision making. Journal of Economic Perspectives, 19(4), $25-42$.

Ho, T.-H., Camerer, C. F., \& Weigelt, K. (1998). Iterated dominance and iterated best response in experimental "P-beauty contests". American Economic Review, 88(4), 947-969.

Holt, C. A., \& Laury, S. K. (2002). Risk aversion and incentive effects. American Economic Review, 92(5), 1644-1655.

Hristova, E., \& Grinberg, M. (2005). Information acquisition in the iterated prisoner's dilemma game: An eye-tracking study. In Proceedings of the 27th annual conference of the cognitive science society (pp. 983-988).

Johnson, E. J., Camerer, C. F., Sen, S., \& Rymon, T. (2002). Detecting failures of backward induction: Monitoring information search in sequential bargaining. Journal of Economic Theory, 104(1), $16-47$.

Knoepfle, D., Wang, J. T., \& Camerer, C. F. (2009). Studying learning in games using eye-tracking. Journal of the European Economic Association, 7(2-3), 388-398.

Manor, B. R., \& Gordon, E. (2003). Defining the temporal threshold for ocular fixation in free-viewing visuocognitive tasks. Journal of Neuroscience Methods, 128(1-2), 85-93. 
Nagel, R. (1995). Unraveling in guessing games: An experimental study. American Economic Review, 85(5), 1313-1326.

Rabin, M. (1993). Incorporating fairness into game theory and economics. American Economic Review, $83(5), 1281-1302$.

Rydval, O., Ortmann, A., \& Ostatnicky, M. (2009). Three very simple games and what it takes to solve them. Journal of Economic Behavior \& Organization, 72(1), 589-601.

Stahl, D. O., \& Wilson, P. W. (1994). Experimental evidence on players' models of other players. Journal of Economic Behavior \& Organization, 25(3), 309-327.

Stahl, D., \& Wilson, P. W. (1995). On players' models of other players: Theory and experimental evidence. Games and Economic Behavior, 10(1), 218-254.

Stewart, N., Gächter, S., Noguchi, T., Mullett, T. L. (2015). Eye movements in strategy choice. Mimeo. http://www.stewart.warwick.ac.uk/publications/papers/Stewart_Gaechter_Noguchi_Mullett_2015. pdf.

Unsworth, N., \& Engle, R. W. (2007). On the division of short-term and working memory: An examination of simple and complex span and their relation to higher order abilities. Psychological Bulletin, 133(6), 1038-1066.

Walsh, W. B., \& Betz, N. E. (1990). Tests and assessment. Englewood Cliffs, NJ: Prentice Hall.

Wang, J. T., Spezio, M., \& Camerer, C. F. (2010). Pinocchio's pupil: Using eyetracking and pupil dilation to understand truth-telling and deception in games. American Economic Review, 100(3), 984-1007.

Wechsler, D. (1987). Manual for the Wechsler memory scale-revised. San Antonio, TX: The Psychological Corporation.

Weizsäcker, G. (2003). Ignoring the rationality of others: Evidence from experimental normal-form games. Games and Economic Behavior, 44(1), 145-171. 